\title{
Class-I MHC-Restricted T-Cell Associated Molecule (CRTAM) Expression in Cerebellum
}

\section{Esther López-Bayghen ${ }^{1 *}$, Karla Pérez-Toledo ${ }^{1,2}$, Leticia Ramírez- Martínez ${ }^{1}$, Jacqueline Robledo ${ }^{1}$, Vianney Ortiz ${ }^{3}$ and Arturo Ortega ${ }^{1}$}

${ }^{1}$ Departamento de Toxicología, Centro de Investigación y de Estudios Avanzados del IPN, Ciudad de México, México

${ }^{2}$ Departamento de Genética y Biología Molecular, Centro de Investigación y de Estudios Avanzados del IPN, Ciudad de México, México

${ }^{3}$ Departamento de Biomedicina Molecular, Centro de Investigación y de Estudios Avanzados del IPN, Ciudad de México, México

*Corresponding Author: Esther López-Bayghen, Departamento de Toxicología, Centro de Investigación y de Estudios Avanzados del IPN, Ciudad de México, México.
Received: May 23, 2020;

Published: June 08, 2020

(C) All rights are reserved by Esther LópezBayghen., et al.

\begin{abstract}
The class-I MHC-restricted T-cell associated molecule (CRTAM), an activation molecule expressed in activated Natural Killer cells (NKT) and CD8+ T cells, is highly expressed in the cerebellar cortex, both in neurons and glial cells. During cerebellar development, granule cell migration over Bergmann glia cells depends on neuronal/glia glutamatergic signaling, suggesting a regulated cell-cell interaction process, opening the possibility of CTRAM and Nectin-like 2 involvement. Through the use of the well-established model of chicken cerebellar Bergmann glial cells and a wound/healing assay, we describe herein that glutamate represses CRTAM expression, enhancing migration. These results reveal an unexpected function of CTRAM in the cerebellum.
\end{abstract}

Keywords: CRTAM; Nectin-Like 2; Cell Migration; Glutamate Receptors; Bergmann Glial Cells

\section{Abbreviations}

AP-1: Activator Protein 1; Bis I: Bis-indolylmaleimide I; BGC: Bergmann Glial Cells; CADM1- 4: Cell Adhesion Molecule Genes; CNRS1-

3: Three Conserved Non-Coding Region Sequences; CNS: Central Nervous System; CRTAM: Class-I MHC-Restricted T-Cell Associated Molecule; GCPs: Granule Cell Progenitors; Glu: Glutamate; IFN-团: Interferon-gamma; IGL: Inner Granule Cell Layer; IL-22: Interleucine 22; JNK: c-Jun N-Terminal Kinase; KA: Kainic Acid; ML: Molecular Layer; Necl-2: Nectin like-2; NMDA: N-Methyl-D-Aspartate; NKT: Natural Killer T Cells; PCL: Purkinje Cell Layer; SynCAMs: Nectin-Like Molecules or Synaptic Cell Adhesion Molecules; TCR: T Cell Receptor; ZEB1: zinc finger E-box binding protein.

\section{CRTAM in cell migration at cerebellum}

The class-I MHC-restricted T-cell associated molecule (CRTAM), or CD355, a member of the immunoglobulin superfamily, is an activation molecule predominantly expressed in activated human Natural killer T cells (NKT) and CD8+ T cells [1-3]. CRTAM expression has been associated with IFN- $\gamma$ production in CD8+ T cells and with NK cell cytotoxicity towards a tumor cell line [4]. A small population of IFN- $\gamma$ - and IL-17-producing CD4+ T cells also express CRTAM upon activation. A transient CRTAM expression in CD8+ T cells, NK and NKT cell following activation has been reported [2-6]. The involvement of CTRAM in the establishment of T helper 1 cells, Th1 and Th17 proinflammatory responses have also been documented [7]. Interaction of CRTAM with its ligand, Nectinlike 2 (Necl-2), enhances IFN- $\gamma$ and IL22 (interleukin 22) production in CD8+ T cells and cytotoxicity of NK against tumors $[4,6]$.

CRTAM expression in cell lymphocytes is triggered by cell activation, specifically for TCD8+, NK, and TCD4+ cells. CRTAM plays multiple and diverse roles in lymphocyte function. It promotes TCD8+ cell adhesion and retention within the lymph node and induces a late phase of cell polarity during activation [7].

The ablation of CRTAM has been reported. CRTAM -/- cells show increased $\mathrm{T}$ cell receptor (TCR)-mediated proliferation and reduced IFN gamma and IL22 production [7]. Moreover, these cells exhibit defects in cell polarity, a function that has been associated to its Postsynaptic density protein (PSD95), Drosophila disc large 
suppressor (Dig1), and zonula occludens-1 protein (ZO-1) (PDZ)binding domain $[7,8]$. Blocking CRTAM-Necl2 interaction impairs thymus growth, impacting structural maintenance of thymic lobes [9]. CRTAM KO mice exhibit reduced protective immunity against viral infection and impaired autoimmune diabetes induction in vivo [10]. Needless to point out, CRTAM signaling is required for the induction of CD4+ CTL and IFN- $\gamma$ production.

CRTAM is an evolutionary conserved receptor-like molecule; it exhibits two Ig domains in the extracellular region, with five cysteine residues, four of which participate in the formation of the Iglike domains [3].

In the mouse, CRTAM mRNA is detectable in spleen, brain, and testis, and in humans, it is present in the spleen, thymus, intestine, lymph nodes, lungs, testis, ovaries, and colon $[2,3]$.

The rapid, swift, and robust induction of CRTAM expression in T and NK cells suggests that its function is time-dependent, and thus its expression must be tightly regulated. CRTAM gene expression involves a transcriptional mechanism. The regulatory elements and the signaling pathway that regulates CRTAM gene activation has been analyzed. A web-based global alignment tool was used to identify the potential regulatory factors that control CRTAM gene expression, within approximately $6 \mathrm{~kb}$ of the 5 -flanking region of human, mouse, rat, and dog CRTAM gene. Three conserved noncoding region sequences (CNRS1-3) spread along $3 \mathrm{~kb}, 5^{\prime}$ from the ATG translation codon, were described. These CNRS regions shared a significant identity, between $50 \%$ and more than $75 \%$ sequence identity in the more significant $100 \mathrm{bp}$ long elements [11].

The CRTAM gene is controlled by a TCR/JNK (c-Jun N-terminal kinase) dependent signaling pathway, in which the participation of a distal cis-element in the CRTAM promoter recognized by the Activator Protein 1 (AP-1) transcription factor is plausible [11]. Recent studies show that CRTAM is a molecular target for ZEB1 (zinc finger E-box binding protein), a homeodomain/Zn finger transcription factor. Overexpression of ZEB1 represses CRTAM promoter activity, as well as endogenous CRTAM levels in human T cells [12].

Tissue-specific regulatory mechanisms for CRTAM gene expression are expected; the constitutive expression CRTAM protein in cerebellar Purkinje cells strongly supports this interpretation. Interestingly, CRTAM is highly expressed in the cerebellum, and this expression is mainly localized in Purkinje neurons, although it is also present in granule cells [3]. CRTAM/Necl-2 heterodimers may contribute to neuronal interactions, specifically in cell-cell interactions between Purkinje neurons and adjacent cells in the cerebel$\operatorname{lum}[3,6]$.
Nectin-like molecules or synaptic cell adhesion molecules (SynCAMs) are four membrane proteins belonging to the immunoglobulin (Ig) superfamily and encoded by the cell adhesion molecule genes (CADM1-4). SynCAM proteins have an extracellular sequence with three Ig-like domains for adhesive interactions, a single transmembrane region, and a short cytosolic sequence with binding motifs for FERM and PDZ domains [13]. SynCAMs 1-4 function in neurons as synaptic cell adhesion molecules and they are widely expressed throughout the developing of Central Nervous System (CNS) exhibiting a distinct spatiotemporal expression pattern indicating that SynCAM-mediated adhesion may help to spatially define cellular interactions in the brain $[14,15]$.

Differential expression of adhesion molecules contributes to defining topographic maps and neuronal circuitry [16]. SynCAMs exhibit a distinct spatiotemporal expression pattern; this framework is observed in all regions: particularly in the Cerebellum, SynCAMs display highly distinct expression in cerebellar granule and Purkinje cells [15]. Nevertheless, in the developing hippocampus, SynCAMs appear to be expressed predominantly in neurons, and localize to synapses in vitro, consistent with their role as synaptic plasma membrane proteins in the brain [14]

SynCAM 2 expression is pronounced in the Purkinje cell layer and has been detected in cerebellar granule cells. In adults, it appeared uniformly low across all cell populations of the cerebellum. SynCAM 3 was the most prominent family member in the cerebellum; at postnatal day 15 , its expression was higher and limited to granule cells, but it was not detected in the Purkinje cell layer expression at this time point. In the adult mice, the granule cell expression of SynCAM 3 appeared even stronger, and significant expression of SynCAM 3 was additionally observed in Purkinje cells. SynCAM 2 and 3, in conjunction with N-cadherin, have been shown to participate in the formation of synapses in the hippocampus [17] and SynCAM 3 has been localized to non-junctional cell-cell contact sites in neural tissue [18]. In the Cerebellum, SynCAM 4 was the second most prominently expressed family member. Strikingly, at P15, SynCAM 4 showed an expression pattern inverse to that of SynCAM 3; a very high SynCAM 4 expression was evident only in the Purkinje cell layer and this pattern persisted into adulthood with some weak expression by the granule cells [15].

During the development of the CNS, synapse formation is required for wiring neuronal circuits and continues to remodel adult networks [13]. SynCAMs can be the primary activator of synapse formation as they participate in the assembly of specialized cellcell junctions such as neuronal synapses. SynCAM 1 (Nectl-2) can promote synapse formation and restrict synaptic plasticity to regulate the wiring and remodeling of neuronal circuits. Recently, a 
significant SynCAM1/Nectl-2 contribution to excitatory synapse number in the developing and adult brain was shown [19]. SynCAM1/Nectl-2 is expressed throughout the central and peripheral nervous system, with the highest levels of SynCAM1/Nectl-2 detected in the cerebellum. The coordinate expression of these two molecules supports the functional association of CRTAM and SynCAM1/Nectl-2, suggesting strongly that these molecules play a role in the cellular interactions of the cerebellum and an essential role in the function of Purkinje neurons [3]. Analysis of the SynCAMadhesion molecule expression profile reported a weak expression of SynCAM1/Nectl-2, and it was also detected in the Purkinje and granule cell layer at both P15 and adult stages. CRTAM interacts with SynCAM1/Nectl-2 suggesting that the expression pattern can be similar to this molecule in the different compartments of the brain.

\section{Cerebellum development and glutamate regulation}

The cerebellum is structure critically involved in motor control. The cerebellar cortex is composed of a laminar structure of three layers. The first layer (outside), is an outer molecular layer (ML) that contains granule neuron axons, Purkinje cell dendrites, Bergmann glial fibers, basket cells, and stellate cells; in the second layer (middle), a Purkinje cell layer (PCL) that contains the Purkinje cells and Bergmann glial bodies; and finally, an inner granule cell layer (IGL) that contains the granule cells and Golgi cells [20-22]. To establish the correct cerebellum structure, the development of the laminar cerebellum requires the coordinated interaction between three main cell types, the Purkinje cells, glial cells, and granule cells.

During cerebellar development, neuronal differentiation involves migration, directional growth, synaptogenesis, and selective survival. Neuronal migration during brain development was first proposed for cerebellar granule cells, assumed that granule cells translocate from their birthplace to their final destination, where they reside during the adult life which may indicate that the migration of immature neurons is essential for the formation of normal neuronal cytoarchitecture [23]. Radial glia is the leading cellular population required for the migration of several neuronal progenitors, like Purkinje cell progenitors [24], while Bergmann glia is essential to guide the migration of postmitotic GCPs in postnatal cerebellar development [25]. After birth, cerebellum development continues: between the second and fourth days postnatal (P2-P4), several signaling pathways promote granule cell progenitors (GCPs) proliferation [26]. Concomitantly, between birth and the end of the second postnatal week, GCPs exit the cell cycle and move into the inner regions of the EGL where they extend parallel fibers and migrate along the radial fibers of Bergmann glial cells
(BGC) to a position under the Purkinje cells, where they form the inner granule layer (IGL) [21]. A number of regulatory factors including neurotransmitters as glutamate (Glu), neuropeptides like somatostatin, glycoproteins, growth factors as the brain-derived nerve factor or the insulin growth factor-1, cytokines (stromal cell-derived factor-1), glycosphingolipids (9-0-acetyl ganglioside3, 9-0-acetyl GD3) and phospholipids (platelet-activating factor) facilitate or exert a direct stimulatory activity or an immediate inhibitory effect on the migration of interneurons in different cortical layers of the postnatal Cerebellum [23]. Glutamate (Glu) is the main excitatory neurotransmitter in the Central Nervous System of most vertebrates [27], acting through the activation of ionotropic (ligand-gated ion channels) and metabotropic (G-protein coupled) receptors, expressed in neurons and glial cells [28]. A membrane to nucleus signal transduction cascades that regulate gene expression at the transcriptional level has been described in BGC exposed to Glu [29-31]. Glutamate signaling is present in the developing cerebellum, where granule cells migrate from the germinal external granular layer along the previously established Bergmann glial fibers [32]. Disruption of glial/neuronal Glu signal transduction, through inhibition of the Glu receptors and transporters, impairs granule cell migration, synaptogenesis, and proper cerebellar architecture [33]. It is quite evident that Glu-driven differential gene expression patterns in neurons and glial cells are the molecular frameworks of this sophisticated developmental program [34].

Glutamate down-regulation of CRTAM and nectin-like 2 inhibits Bergmann glial cell migration in vitro

Although some reports have shown that CRTAM is expressed in the cerebellum, more studies are needed for the understanding of its role in the brain. Among the various proteins involved in cerebellar development, CRTAM is of particular interest, given the fact that its transcription is controlled by AP-1 [11], and that is highly expressed in the Cerebellum [3]. AP-1 is a common mediator of Glu-dependent transcriptional response in BGC [35-37]. Taking advantage of the well-characterized BGC culture, we sought to gain insight into Glu-mediated cell migration, concerning CRTAM and Necl-2 and describe in the next sections, the glutamate-mediated downregulation of CRTAM and Nectin-like 2 expression, which impedes cell migration.

To look for membrane proteins involved in cell-cell interactions during cerebellar development and the effect of Glu, wound healing assays were performed on confluent BGC (monolayers were wounded and followed for $48 \mathrm{~h}$ ), which are widely used to evaluate cell migration [38]. While the control demonstrated typical recovery, treatment with $1 \mathrm{mM}$ Glu led to a diminished time-dependent migratory capacity of the BGC $\left(\mathrm{p}^{*}<0.05\right)$ (Figure $\left.1 \mathrm{~A}\right)$. 

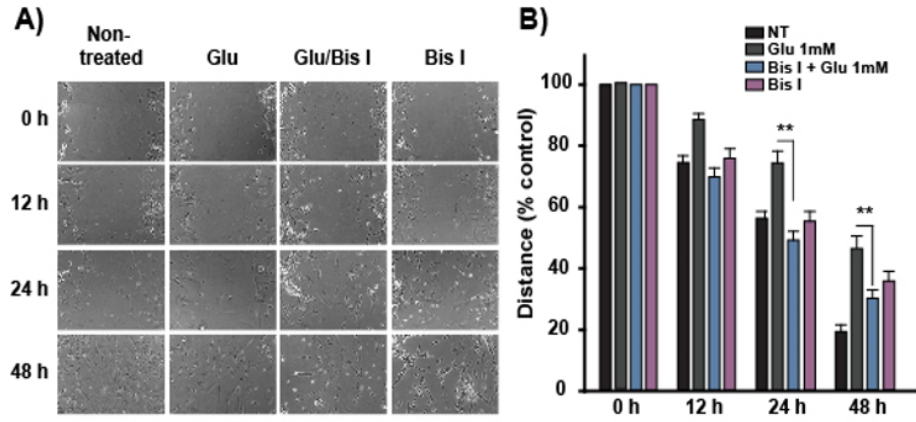

C)

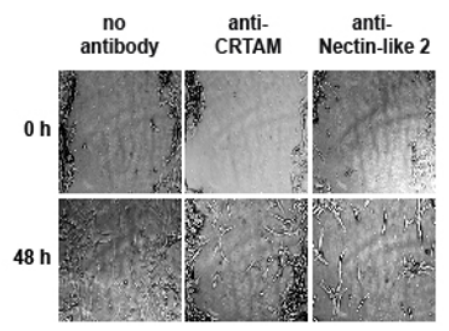

D)

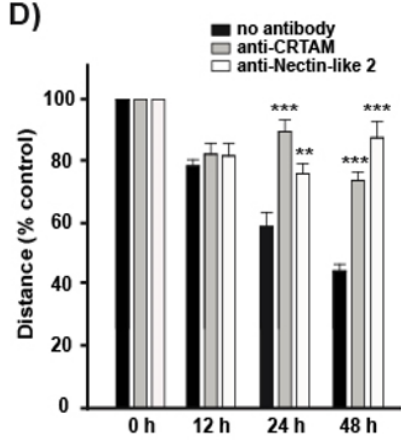

Figure 1: Glutamate inhibits Bergmann glia migration. CRTAM and Nectin-like 2 co-localization affect cell migration in Bergmann glial cells. Primary cultures of BGCs prepared from 14-day-old chick embryos as (Ortega., et al. 1991) were plated in 12-well plastic cell culture plates ( 1 x 106) in Dulbecco's modified Eagle's medium (DMEM) containing 10\% fetal bovine serum (FBS), $2 \mathrm{mM} \mathrm{glutamine} \mathrm{and} 50 \mu \mathrm{g} / \mathrm{ml}$ gentamicin, and maintained at $37^{\circ} \mathrm{C}$ with $5 \% \mathrm{CO} 2$. At $85 \%$ confluence (3 - 4 days), monolayers were switched to low-serum DMEM media $(0.5 \% \mathrm{FBS})$ for $8 \mathrm{~h}$ and treated with the PKC inhibitor Bis I $(1 \mu \mathrm{M})$ for 30 minutes in fresh DMEM media before Glutamate (Glu, $1 \mathrm{mM})$ treatment (A and B) or pretreated $2 \mathrm{~h}$ with anti-CRTAM (1:100) and anti-Necl-2 (1:100) antibodies (C and D). To assay cell migration, BGC monolayers were immediately wounded with a wide pipette tip to create a cleared line; cell migration was monitored at $0,12,24$, and 48 h. The wound closure process was photographed at 0,12, 24, and 48 h using a Nikon Eclipse TS100 Microscope with a Premiere MA88500 camera. (B and D) To determine the distance, pixels in the denuded area were quantified for each condition at the indicated times using the Adobe Photoshop software. Data are expressed as the mean \pm standard error of at least three independent experiments. A oneway ANOVA and a post hoc Student- Newman-Keuls test analysis were used to determine which conditions were significantly different (Prism, GraphPad Software) (**, p $<0.01 ; * * *, p<0.001)$.

Taking into consideration that ionotropic Glu receptors of the AMPA subtype expressed in this cell system are devoid of the ionotropic receptor AMPA type subunit 2, and therefore are $\mathrm{Ca}^{2+}$-permeable $[39,40]$, a possible link to PKC activation was hypothesized [41]. Pretreatment with Bisindolylmaleimide (BisI), a PKC inhibitor, attenuated Glu-mediated migratory inhibition, and treatment of BisI alone demonstrates no significant effect on cell migration when compared to control (Figure 1B), suggesting that glutamate regulates the migratory capacity of BGC through PKC activation.

After confirming CRTAM, and Nectin-like 2 expression with PCR (Figure 2A), we assessed if these receptors affect cell migration. Antibodies for CRTAM and Nectin-like 2 were enough to inhibit
Bergmann glia migration as treated monolayers showed a 2.5 to 3.3-fold decrease in migration, respectively when compared with control ( $\mathrm{p}^{* *}<0.01$, Figure 1C and 1D).

The most abundant glutamatergic synapse in the vertebrate brain is between the Purkinje cell dendrites and the axonal terminals of the granule cells (parallel fibers), and that these synapses are entirely encompassed by Bergmann glial cells [42]. There is a significant expression of CRTAM in Purkinje cells [3] that prompted us to consider a possible role for Glu in controlling the expression of CRTAM and Nectin-like 2. It has been previously demonstrated that in cultured Bergmann glial cells, Glu triggers a membrane-tonucleus signaling cascade involved in transcriptional repression of 
the chglast gene [43], which involved PKC activation. As PKC activation is associated with AP-1, in silico analysis of the CRTAM putative promoter region highlighted an AP-1 DNA binding site [11], which is also a mediator of glutamate transcriptional responses in Bergmann glia [35,43]. When Bergmann glia cells monolayers were exposed to glutamatergic ligands, Kainic acid (KA) and Nmethyl-D-aspartate (NMDA), a decreased expression of CRTAM, and Nectin-like 2 mRNAs were recorded, like treatment with Glu (Figure 2). Interestingly, this effect was mimicked by KA, as well as by NMDA, reflecting the involvement of AMPA, and NMDA subtypes of receptors, both linked to the $\mathrm{Ca}^{2+}$ influx and $\mathrm{Ca}^{2+} /$ diacylgliceroldependent PKC activation [29,44].

Interestingly, pre-treatment with BisI attenuated KA, and NMDA's effect on expression ( $\left.p^{*}<0.05\right)$. Tetradecanoyl phorbol ac- etate (TPA), a PKC activator, also decreased CRTAM and Nectin-like 2 levels, like treatment with glutamate, which was lost with BisI pre-treatment (Figure 2). Therefore, we examined if a PKC activator could mimic this effect, and reciprocally, a PKC inhibitor could block it. A detailed analysis of both promoters in the context of our cultured cells is needed, and it is currently in progress in our laboratory, focusing on delineating the signaling triggered by glutamate that represses crtam gene transcription.

As determined with immunofluorescence, the treatment with either Glu or TPA resulted in decreased expression and loss of colocalization of CRTAM and Nectin-like 2 proteins (Figure 3); however, this effect was lost when pretreated with BisI. Thus, glutamate signaling, through a PKC dependent pathway, negatively regulates CRTAM and Nectin-like 2 expression, and by these means glial cell migration.
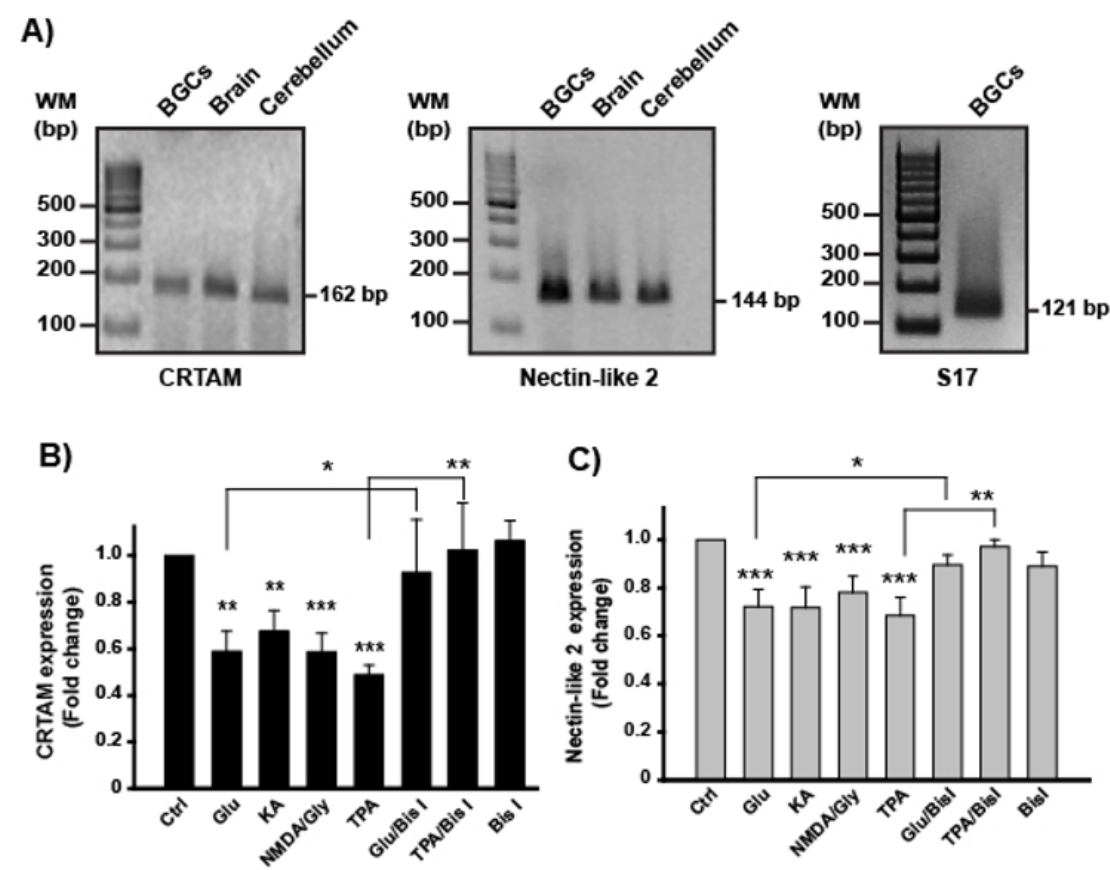

Figure 2: Glutamatergic stimuli reduced the expression of CRTAM and Nectin-like 2 via PKC. Total RNA from the brain, cerebellum (rat tissue, positive controls) and BGCs was extracted using the Trizol protocol (manufacturer's instructions). qRT-PCR was performed with the KAPA SYBR FAST One-Step qRT-PCR Kit, to assess the expression of CRTAM, Necl-2, and S17. (A) Representative qRT-PCR amplified products of CRTAM and Necl-2 in the rat brain, cerebellum and BGCs; S17 chick ribosomal mRNA is the internal control. To determine CRTAM and Necl-2 regulation, BGCs were initially pretreated with Bis I $(1 \mu \mathrm{M})$ for 30 minutes or not, followed by treatment with glutamate (Glu, $1 \mathrm{mM})$, kainic acid (KA, $500 \mu \mathrm{M})$, NMDA $(500 \mu \mathrm{M})+$ Glycine $(0.01 \mathrm{mM})$, TPA (100 nM) or DMSO (control) for $2 \mathrm{~h}$. After 24 h, RNA was isolated, and relative mRNA expression for CTRAM (B) and Nectin-like 2 (C) was determined. The relative abundance of mRNA was expressed as sample versus control in comparison to S17 mRNA, calculated using the 2- $\Delta \Delta \mathrm{Ct}$ method. Data are expressed as the mean \pm SE of at least three independent experiments by triplicate. A one-way ANOVA was performed and a post hoc Student- Newman-Keuls test analysis were used to determine which conditions were significantly different (Prism, GraphPad Software)

$$
(*, \mathrm{p}<0.05 ; * *, \mathrm{p}<0.01 ; * *, \mathrm{p}<0.001) \text {. }
$$




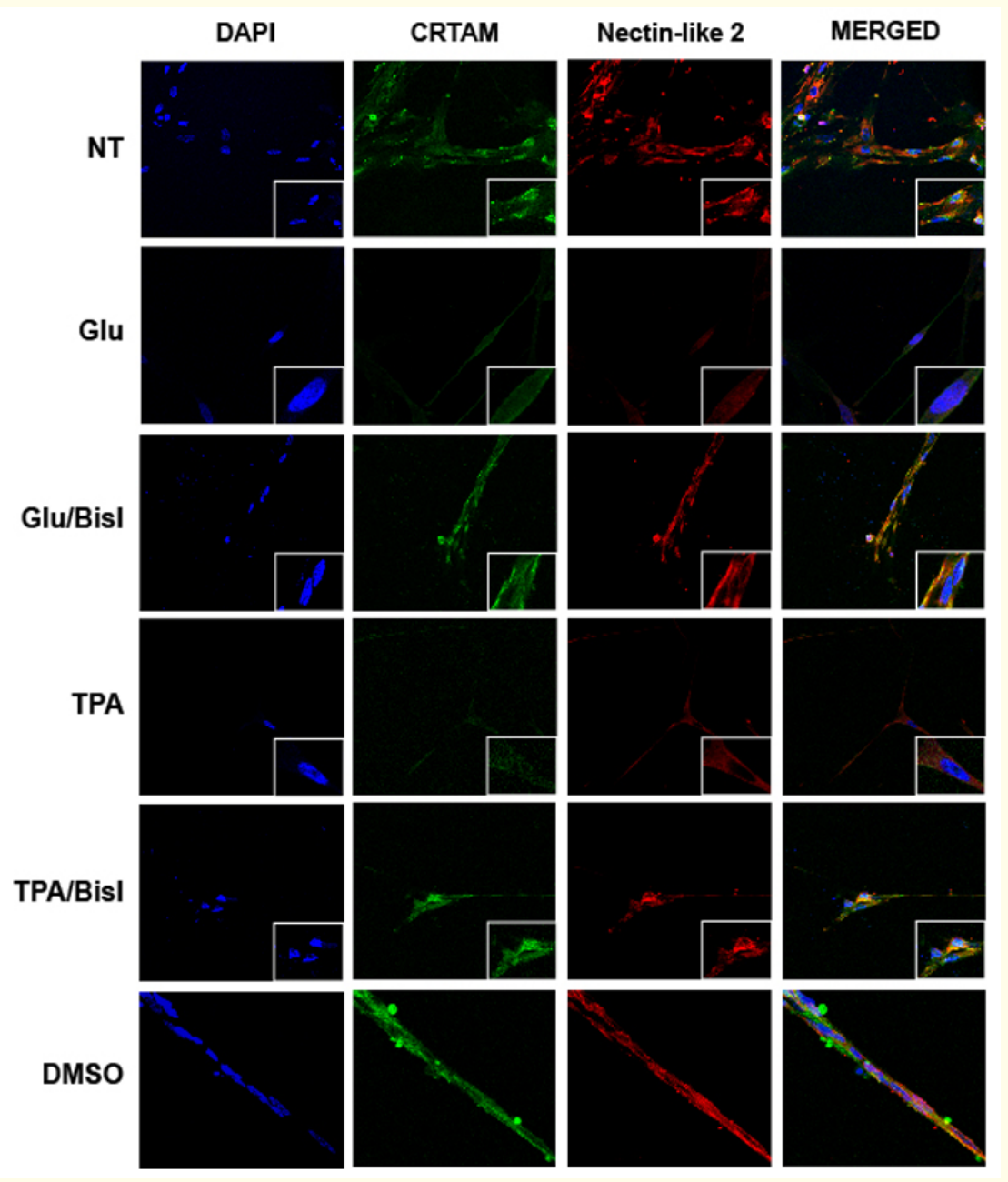

Figure 3: Glutamate downregulates CRTAM and Nectin-like 2 protein expression and co-localization. BGCs were grown in eight-well Lab-Tek Chamber Slides to explore CRTAM and Nectin-like 2 protein localization, and stimulated with Glutamate (Glu, 1 mM), TPA (100 $\mathrm{nM}$ ), or DMSO (control) for two hours. Bis I $(1 \mu \mathrm{M})$ was added 30 min before treatment where indicated. Cells were fixed $24 \mathrm{~h}$ after glutamate or TPA treatment with 3.4\% of paraformaldehyde and immunostained with antibodies anti-CRTAM (1:100, green) or anti-Nectin-like 2 (1:100, red), nucleus stained with DAPI (blue). Fluorescence was examined using a Leica confocal microscope (Leica TCS-SPE, oil 63x objective (zoom 1)).

\section{Conclusion}

Figure 4 summarizes the Glu-dependent transcriptional control involved in the ability of glial cells to guide the migration. Glu signaling, through a PKC dependent pathway, negatively regulates CRTAM and Nectin-like 2 gene and protein expression, thus affecting cell migration capacity. CRTAM and Nectin-like 2 expression and localization are affected by glutamatergic signaling, strengthening the notion of a pivotal role of glial cells in the development and structural arrangement of the cerebellar molecular layer.

\section{Acknowledgments}

This project was supported in part by grants from Conacyt 255087 to AO and 155605 to ELB. The authors acknowledge the secretarial assistance of Ing. Lucero Cervantes Pozos.

\section{Conflict of Interest}

The authors declare no financial interest or any conflict of interest. 


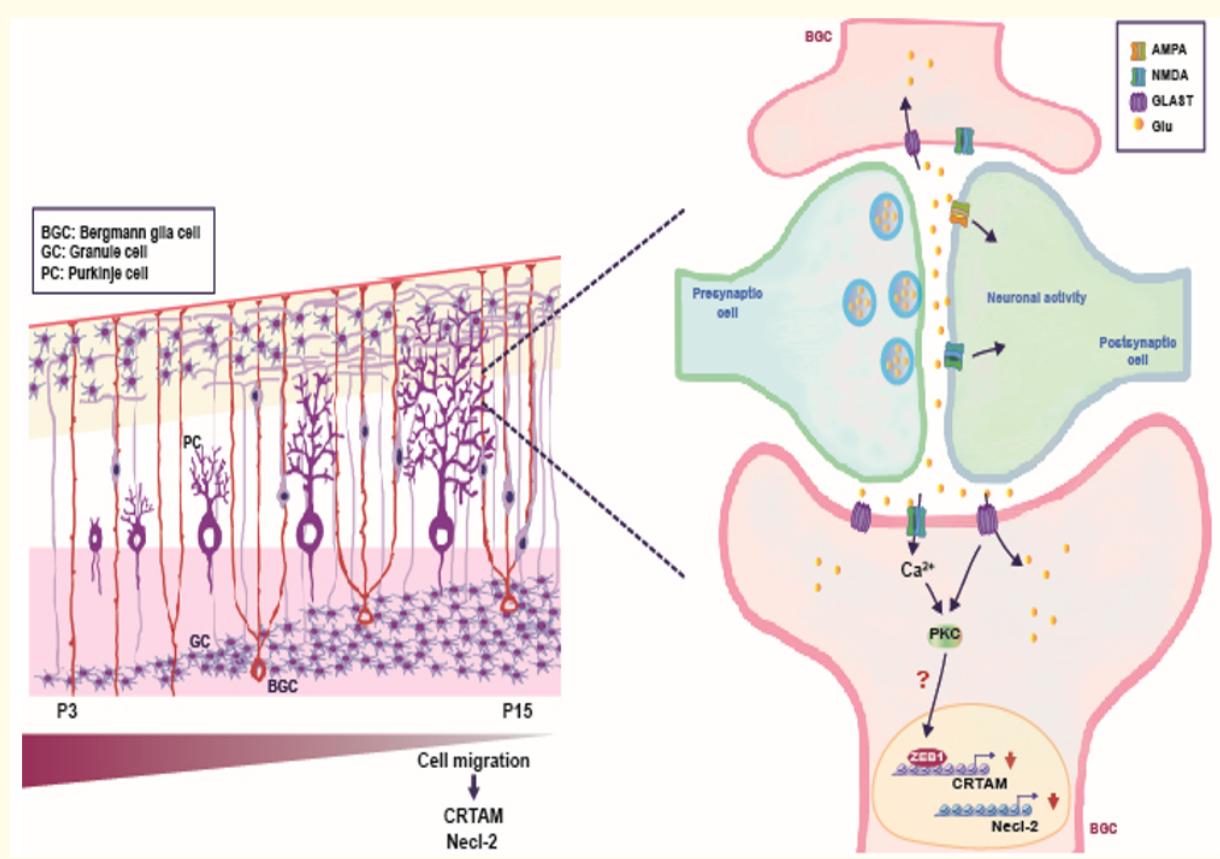

Figure 4: Model for CRTAM regulation in the cerebellum. CRTAM and Necl-2 are downregulated by glutamate via PKC in Bergmann glial cells. During cerebellum development, the migration cells from the external granule layer (EGL) to the internal granule layer (IGL) is the dominant process. In the late development period (P15-20), migration is bringing down, accompanied by a decrease of CRTAM and Necl-2 expression in Bergmann glial cells (BGC). Glutamatergic synapses now are the preeminent function between Purkinje cells (PC) and BGCs in the mature cerebellum, the glutamate (Glu) release by PC is capture by BGC.

\section{Bibliography}

1. Patino-Lopez G., et al. "Human class-I restricted T cell associated molecule is highly expressed in the Cerebellum and is a marker for activated NKT and CD8+ T lymphocytes". Journal of Neuroimmunology 171.1-2 (2006): 145-155.

2. Kennedy J., et al. "A molecular analysis of NKT cells: identification of a class-I restricted T cell-associated molecule (CRTAM)". Journal of Leukocyte Biology 67.5 (2000): 725-734.

3. Patino-Lopez G., et al. "Human class-I restricted T cell associated molecule is highly expressed in the Cerebellum and is a marker for activated NKT and CD8+ T lymphocytes". Journal of Neuroimmunology 171.1-2 (2006): 145-155.

4. Boles KS., et al. "The tumor suppressor TSLC1/NECL-2 triggers NK-cell and CD8+ T-cell responses through the cell-surface receptor CRTAM". Blood 106.3 (2005): 779-786.

5. Arase N., et al. "Heterotypic interaction of CRTAM with Necl2 induces cell adhesion on activated NK cells and CD8+ T cells". International Immunology 17.9 (2005): 1227-1237.
6. Galibert L., et al. "Nectin-like protein 2 defines a subset of Tcell zone dendritic cells and is a ligand for class-I-restricted T-cell-associated molecule". Journal of Biological Chemistry 280.23 (2005): 21955-21964.

7. Yeh JH., et al. "Regulation of a late phase of $\mathrm{T}$ cell polarity and effector functions by Crtam”. Cell 132.5 (2008): 846-859.

8. Tonikian R., et al. "A specificity map for the PDZ domain family". PLoS Biology 6.9 (2008).

9. Medina-Contreras 0., et al. "Role of CRTAM during mouse early T lymphocytes development". Developmental and Comparative Immunology 34.2 (2010): 196-202.

10. Takeuchi A., et al. "CRTAM confers late-stage activation of CD8+ T cells to regulate retention within lymph node". Journal of Immunology 183.7 (2009): 4220-4228.

11. Valle-Rios R., et al. "Characterization of CRTAM gene promoter: AP-1 transcription factor control its expression in human T CD8 lymphocytes". Molecular Immunology 46.16 (2009): 3379-3387. 
12. Rojas-Marquez C., et al. "CRTAM is negatively regulated by ZEB1 in T cells". Molecular Immunology 66.2 (2015): 290-298.

13. Biederer T. "Bioinformatic characterization of the SynCAM family of immunoglobulin-like domain-containing adhesion molecules". Genomics 87.1 (2006): 139-150.

14. Fogel AI., et al. "SynCAMs organize synapses through heterophilic adhesion". The Journal of Neuroscience 27.46 (2007): 12516-12530.

15. Thomas LA., et al. "Expression and Adhesion Profiles of SynCAM Adhesion Molecules Indicate Distinct Neuronal Functions". The Journal of Comparative Neurology 510.1 (2008): 47-67.

16. Benson DL., et al. "Molecules, maps and synapse specificity". Nature Reviews Neuroscience 2.12 (2001): 899-909.

17. Mizoguchi A., et al. "Nectin: an adhesion molecule involved in formation of synapses". Journal of Cell Biology 156.3 (2002): 555-565.

18. Kakunaga S., et al. "Nectin-like molecule-1/TSLL1/SynCAM3: a neural tissue-specific immunoglobulin-like cell-cell adhesion molecule localizing at non-junctional contact sites of presynaptic nerve terminals, axons and glia cell processes". Journal of Cell Science 118.6 (2005): 1267-1277.

19. Robbins EM., et al. "SynCAM 1 Adhesion Dynamically Regulates Synapse Number and Impacts Plasticity and Learning". Neuron 68.5 (2010): 894-906.

20. Voogd J and M Glickstein. "The anatomy of the Cerebellum". Trends in Cognitive Sciences 2.9 (1998): 307-313.

21. Roussel MF and ME Hatten. "Chapter 8 - Cerebellum: Development and Medulloblastoma, in Current Topics in Developmental Biology, M.A. Dyer, Editor, Academic Press (2011): 235-282.

22. Xu H., et al. "Bergmann Glia Function in Granule Cell Migration During Cerebellum Development". Molecular Neurobiology 47.2 (2013): 833-844.

23. Galas L., et al. "Postnatal Migration of Cerebellar Interneurons”. Brain Sciences 7.6 (2017).

24. Morales D and ME Hatten. "Molecular markers of neuronal progenitors in the embryonic cerebellar anlage". The Journal of Neuroscience 26.47 (2006): 12226-12236.
25. Hatten ME. "New directions in neuronal migration". Science 297.5587 (2002): 1660-1663.

26. Wechsler-Reya RJ and M Scott. "Control of neuronal precursor proliferation in the Cerebellum by Sonic Hedgehog". Neuron 22.1 (1999): 103-114.

27. Fonnum F. "Glutamate: a neurotransmitter in mammalian brain". Journal of Neurochemistry 42.1 (1984): 1-11.

28. Hollmann M and S Heinemann. "Cloned glutamate receptors". Annual Review of Neuroscience 17 (1994): 31-108.

29. Lopez-Bayghen E and A Ortega. "Glial glutamate transporters: new actors in brain signaling”. IUBMB Life 63.10 (2011): 816823.

30. Martinez-Lozada Z., et al. "Signaling through EAAT-1/GLAST in cultured Bergmann glia cells". Neurochemistry International 59.6 (2011): 871-879.

31. Mendez-Flores OG., et al. "Coupling of glutamate and glucose uptake in cultured Bergmann glial cells". Neurochemistry International 98 (2016): 72-81.

32. Rakic P. "Neuron-glia relationship during granule cell migration in developing cerebellar cortex. A Golgi and electonmicroscopic study in Macacus rhesus". Journal of Comparative Neurology 141.3 (1971): 283-312.

33. Komuro $\mathrm{H}$ and Rakic. "Modulation of neuronal migration by NMDA receptors”. Science 260.5104 (1993): 95-97.

34. Lyons MR., et al. "The transcription factor calcium-response factor limits NMDA receptor-dependent transcription in the developing brain". Journal of Neurochemistry 137.2 (2016): 164-176.

35. Aguirre A., et al. "Glutamate regulates kainate-binding protein expression in cultured chick Bergmann glia through an activator protein-1 binding site". Journal of Biological Chemistry 275.50 (2000): 39246-39253.

36. Aguirre A., et al. "Glutamate-dependent transcriptional regulation of the chkbp gene: signaling mechanisms". Journal of Neuroscience Research 70.1 (2002): 117-127.

37. Lopez-Bayghen E and A Ortega. "Glutamate-dependent transcriptional regulation of GLAST: role of PKC". Journal of Neurochemistry 91.1 (2004): 200-209.

38. Ascione F., et al. "Comparison between fibroblast wound healing and cell random migration assays in vitro". Experimental Cell Research 347.1 (2016): 123-132. 
39. Müller F., et al. "Glutamate receptor expression in the rat retina". Neuroscience Letters 138.1 (1992): 179-182.

40. López T., et al. "AMPA/KA receptor expression in radial glia". Neuroreport 5.4 (1994): 504-506.

41. Cid ME and A Ortega. "Glutamate stimulates [3H]phorbol 12,13-dibutyrate binding in cultured Bergmann glia cells". European Journal of Pharmacology 245.1 (1993): 51-54.

42. Somogyi P., et al., "Subcellular localization of benzodiazepine/ GABAA receptors in the Cerebellum of rat, cat, and monkey using monoclonal antibodies". Journal of Neuroscience 9.6 (1989): 2197-2209.

43. Rosas S., et al. "Glutamate-dependent transcriptional regulation of GLAST/EAAT1: a role for YY1". Journal of Neurochemistry 101.4 (2007): 1134-1144.

44. Gallo V and CA Ghiani. "Glutamate receptors in glia: new cells, new inputs and new functions". Trends in Pharmacological Sciences 21.7 (2000): 252-258.

\section{Assets from publication with us}

- Prompt Acknowledgement after receiving the article

- Thorough Double blinded peer review

- Rapid Publication

- Issue of Publication Certificate

- High visibility of your Published work

Website: www.actascientific.com/

Submit Article: www.actascientific.com/submission.php

Email us: editor@actascientific.com

Contact us: +919182824667 